Amongst the predisposing causes of appendicitis age is acknowledged to be an important factor, and as the activity of lymphoid tissue is greatest during early life its liability to inflammation in young subjects is not to be wondered at. Abdominal pain in children is frequently localised in the right iliac fossa, and it is probable that repeated slight inflammatory attacks of appendicitis may impair the function of the lymphoid tissue and predispose to the more serious forms of the disease. A similar tendency is observed in connexion with the tonsils, which are frequently found to be much enlarged and indurated, and yet the preceding inflammatory attacks may have been so slight as almost or entirely to have escaped observation. Sir Dyce Duckworth ${ }^{14}$ finds that cold is frequently the immediate cause of appendicitis. Numerous cases have been recorded in which exposure or violent exercise in apparently healthy individuals was the immediate precursor of an attack. As the appendix is an extremely well protected organ as regards external influences, it seems natural to assume that in such cases, as in rheumatism generally, the illness is determined by a general chill which manifests itself locally in a susceptible organ. 'Talamon has observed that heavy eaters are liable to appendicitis, and to this cause he traces the frequency of the affection in England and in America. He says that an indigestible meal is often quickly followed by an attack of acute appendicitis, and this sequence of events is explained by the induction of increased intestinal movements, which drive a fæcal mass into the appendix. In fact, according to his theory there exists an unfortunate class of individuals who possess a cæcum always ready to discharge a fæcal concretion, and an appendix always ready to receive it, but unwilling to part with it. On the other hand, it may be pointed out that a tendency to rheumatism in those who live freely is well known, and an attack of gout or rheumatism frequently follows a heavy meal owing to the absorption of the noxious products of incomplete or perverted digestion. The local manifestations in such cases will vary in different individuals, but in some they would appear to take the form of appendicitis. As regards the clinical signs and symptoms of appendicitis, it is well known that these, as in the case of tonsillitis, may at first point to constitutional disturbance and that there may be nothing to direct attention to the appendix for one or two days. Thus, malaise, headache, nausea, sickness, general aching pains, pyrexia, loss of appetite and furred tongue, constipation or diarrhoea are frequently found to precede any pain or discomfort in the right iliac region, and these are suggestive of a constitutional dyscrasia as the primary disease. Dr. Kelynack ${ }^{15}$ has recorded the case of a medical student who was suddenly seized with an acute illness of which the only definite local sign was tonsillitis, and diphtheria was suspected. On the following day abdominal symptoms appeared, and he died three days later from gangrenous appendicitis and acute suppurative peritonitis. It seems only natural to conclude that the appendix must have been weakened by some previous attacks, and the simultaneous onset of tonsillitis points to a constitutional cause. I have already referred to the occurrence of slight attacks of appendicitis in childhood, and I believe that these are much more frequent than is generally supposed. Such attacks are not considered worthy of more than domestic treatment, and just as rheumatism is ignored under the familiar name of "growing pains," so an attack of appendicitis is regarded as merely a form of "stomachache." The relapsing or recurrent form of appendicitis is characterised by a series of attacks repeated at irregular intervals, and tending in some cases to a condition of chronic ill-health. The relapses are extremely difficult to explain on any theory of local mechanical causation, and it is in this class of cases more especially that Talamon's theory of fæcal concretions appears to me to fail completely. on the other hand, if a constitutional disease such as rheumatism is present, and if it manifests itself by an attack of inflammation in the appendix, we shall at once recognise a liability to the recurrence of inflammation in the same organ. Such a view both coincides with our knowledge of rheumatic disease generally and explains the frequency of recurrent attacks of appendicitis.

If the above statements as to the pathology of simple appendicitis, to which I have been referring more particularly, be accepted the treatment to be adopted will be both constitutional and local. While fully admitting the frequent occurrence, even in previously simple cases, of symptoms demanding immediate surgical interference, I differ entirely from those who hold that all cases of appendicitis onght to be regarded as surgical from the outset. Such an opinion seems to exist in America, and one of the most recent writers on the subject, Dr. G. R. Fowler, ${ }^{16}$ says that "the less medical treatment which cases of appendicitis receivo the better." In this country, however, such extreme views have not yet bcen generally accepted. Mr. Treves, who has shown the value of operative treatment in suitable cases of relapsing typhlitis, states that "the cases adapted for operation are comparatively few, and the simplicity of the surgical treatment has rather cast into the shade the somewhat neglected measures of the physician." 17 In an attack of acute appendicitis in a rheumatic patient the symptoms will often subside rapidly on the administration of full doses of salicylic acid or its compounds. It is also advisable to elear out the bowels by means of two or three grains of calomel, followed by a Seidlitz powder, and to keep up this action by repeated doses of some mild saline. By this means we are able to prevent the accumulation of putrefactive products in. the creum and the consequent risk of septic absorption. Even if diarrhoea is present from the outset, as occasionally happens, it is no contra-indication to the use of mild salines, for the diarrhcea is often caused and maintained by the presence of some irritant in the intestine, and the sooner this is removed and the accompanying putrefactive changes cheeked the better. Opium tends to conceal the symptoms, but if the local pain be excessive a fomentation containing opium and belladonna may be applied over the right iliac region. Rest in bed, warmth, low diet, and the use of local antiphlogistics represent the other details of treatment which. are generally approved of. In the relapsing or more chronic forms of rheumatic appendicitis the life of the patient must be ordered and supervised as in rheumatism generally. Special attention must be paid to the condition of the bowels so as to prevent any accumulation. Some medicine, such as cascara sagrada, is to be taken regularly, and with this may be combined naphthalin (Watson Cheyne), or salol (Treves), or creasote as an intestinal antiseptic. The prolonged use of small doses of salicylate of soda will be found useful in preventing a repetition of the attacks.

old Cavendish-street, $\mathbf{w}$.

\section{A FATAL CASE OF JAUNDICE WITH CEREBRAL SYMPTOMS}

\section{BY SIDNEY H. SNELL, M.D. LOND.,}

LATE HOUSE PHYSICIAN, HOUSE SURGEON, \&C., ONIVERSITY COILEGE HOSPI'TAL, LONDON.

WITH A REPORT ON

\section{THE MICROSCOPICAL APPEARANCES OF THE LIVER.}

BY THE LATE F. V. BUNCH, F.R.C.S. ENG.

FORMERLY SURGICAL REGISTRAR, UNIVERSITY COLLEGE HOSPITAI.

THE patient was a woman aged twenty years. She had: two months previously consulted me for amenorrhoea, which I found to be due to a three and a half months pregnancy. There was a marked phthisical family history, her mother having died from that disease, and since the patient's death her father and a sister have also succumbed to phthisis. In addition, at the apex of the patient's right lung there were signs of some old fibroid trouble. The onset of the present attack was as follows. On Nov. 18th, 1893, the patient seemed to be in her ordinary health and went to London to be married, she being then five and a half months pregnant. She remained at her mother-in-law's house till Nov. 20th, when she returned home feeling a little unwell and com. plaining of indigestion. This feeling continued, and on Nov. 23rd her friends observed that she became slightly jaundiced, vomited occasionally, and refused all food. I saw her first on Nov. 25th in the evening and found her very deeply jaundiced and complaining of severe pain in the right side and general depression. Her manner was a little strange, but she was perfectly sensible; her breathing was. rapid (30), the pulse 70 , and the temperature $99^{\circ} \mathrm{F}$. On examination there was great tenderness over the hepatic area I 
corresponding with the site of the pain, and marked rliminucin in the superficial dulness of this area was inanifest on wercussion. The edge of the liver could not be felt. Splenic Chiness was well marked. Nothing abnornial could be detected elsewhere by physical examination, save the old patch of tubercle bofore allncled to at the apex of the right lung. The footal heart was distinctly audible. the urine was dark in colour and contained bile pigment in abuadance and a trace of albumin. On evaporation to me-fourth of its bulk and the addition of nitric acid no crystals of urea nitrite were obtained, showing the loss of urea. Repeated evaporations and microscopical examinarions of different samples failed at any time to detect the least trace of crystals of either leucin or tyrosin. Owing to pressure of practice, distance from town, \&c., a more comHete analysis of the urine was not possible. On Nov. 26th the patient was clear and sensible, although she had har some delirium during the night. An expression of intense anxiety pervaded the features; the breatling had inoreased in rapidity (37), the tongue was brown and dry, the temperature was $100^{\circ}$, and the pulse 82. Early in the morning the patient had vomited a large quantity of altered blood having the appearance of coffee grouncls. The Wowels had acted twice, the motions being clay coloured. she altered little during that day, but complained of headache and persistently refused nourishment. Towards night mild delirium again came on. On Nov. 27th, at 2 A.M., the patient suddenly became totally blind, retaining only the rerception of strong direct light. Later in the morning the delirium passed off and the patient again became quite rutional, but complained strongly of the pain orer the s apatic region. At this stage her pupils were contracted v) pin point-size. The superficial liver dulness, steadily I. ssening, had by that time entirely disappeared. On the evening of the same day delirium of a much more violent type appeared, and her temperature, taken in the axilla, was $101^{\circ}$, the respiration 38 , and the pulse 140. During that night convulsions supervened, and the' jaundice, which had improved a little, became very much leeper. On the following morning (Nov. 28th) the patient became quite comatose, with now and then an occasional convulsion. The pupils had become, with the onset of the coma, widely dilated. The patient died early on Nov. 29th, ist ten days after the first feelings of malaise and only seven days after the first appearance of jaundice. There were throughout no ascites and no hæmorrhages under the *kin, nor was any itchiness complained of.

With the greatest difficulty I obtained permission for a wartial necropsy. On exposing the liver $I$ found it to be inuch smaller than natural and to be shrunk away towards the back, this accounting for the loss of superficial dulness. $1 t$ weighed, as nearly as I could guess, about thirty ouncesunder two pounds certainly - and was quite firm. The oallbladder was empty. On the surface nunierous yellow patches wuld be seen on the red ground, and on section the organ was dark-red, mottled with yellow. The spleen was enlarged and congested. A few pieces from different parts of the 'iver were secured, and beyond noticing the absence of weritonitis and fluid in the abdomen \&c. I had to make a nurried conclusion owing to the objections of the family. The late Mr. F. V. Bunch--whose recent lamentable death From diphtheria, contracted in the discharge of his duties as surgical rogistrar to University College Hospital, is to be leptored-made the following report on the microscopical appearances of the liver :-

"Pieces of liver were handed to me hardened in alcohol. The capsule was not thickened; it stripped easily, leaving a Nightly granular surface. On the greater part of the cut surface the lobular arrangement of the liver substance was rlearly seen; over a few scattered areas it was, however, *.. Sit. Sections were cut by the paraffin method and stained on the slides, some with Ehrlich's hæmatoxylin, others with gentian violet and eosin. The sections show much connective tissue arranged for the most part in a perilobular manner, and to a less extent in the centres of the lobules and Wetween the columns of liver cells. Different parts show very varying amounts of connective tissue. Where the change is most advanced there are large tracts in which liver substance is only represented by a few islands of Wepatic cells, many of which contain droplets of fat. Granular masses of pigment are also seen. Numerous Franching ducts lined with cubical epithelium are present. Where the process is least advanced the liver lobules are large. Whe centres of the lobules are occupied by a loose-meshed reticulum of connective tissue in which a few liver cells undergoing fatty degencration are enclosed; the change becomes less marked in the zone surrounding this; and in the greater part of the lobule the liver cells are quite healthy. Ducts are plentiful in the perilobular areas. The connective tissue everywhere takes the form of a loose moshwork richly supplied with oval and spindleshaped vuclei. There is, in places, a considerable infiltration with small round cells. There are no hæmorrhages, and crystals of leucin and tyrosin are not seen. The vesscls are healthy. Many examples of division are seen in the nuclei of the liver cells. The appearances of the liver differ from those described in ordinary cases of acute yellow atrophy in the formation of connective tissue; the degeneration of the liver cells is only seen where this connective tissue is formed, and there is therefore a large number of cclls showing no deviation from the normal. The probable pathological process seems to have been an acute formation of fibrous tissue, the destruction of liver substance being secondary."

It will be noticed that this fatal case of jaundice with cerebral symptoms occurred in an unmarried pregnant girl; that is the class of patient attacked in so large a proportion of the cases of that rare disease, acute yellow atrophy of the liver. But although during its clinical course I had but little doubt that such was its nature, on reviewing all its aspects, and especially on studying the microscopic appearance of the diseased organ, it becomes at once evident that striking points of difference exist which mark it out from the usual run of cases of acnte yellow atrophy of the liver. 'Thus the course was much more rapid, there was complete absence of leucin and tyrosin from the urine or from the liver sections, and there was no tendency to hæmornhages (apart from the hæmatemesis) and no sign of abortion. The sudden onset of amaurosis I have failed to find elsewhere recorded. Again, pathologically, the liver, instead of being very flaccid, was actually firmer than natural Microscopically, the appearances were suggestive of an acnte cirrhosis rather than an acute atrophy. I knew the patient's family well, and I made very careful inquiries about her former habits, being specially incited thereto by the warning which Dr. Poore gives in his eloquent lectures on forensic medicine to always be on the qui vive for phosphorus poisoning in cases of acute yellow atrophy. Accordingly, at my very first visit, having managed to send all her friends out of the room, I asked the girl whether she had been taking anything to tamper with herself \&c. To this question she gave a most emphatic denial and with every appearance of truthfulness. Inquiries from the friends elicited that although owing to her pregnant condition she underwent a great deal of mental worry, yet before the visit to London she became very cheerful at the prospect of being married, and, indeed, this event removed the very reason for any question of tampering. One fact was obtained which might have some bearing on the case-i.c., that the patient was in the habit of eating very large quantities (three or four pennyworth daily) of those small pink cachous used for clearing the breath of smell. I endeavoured to obtain some of these from the same source, but failerl. On consulting chemists and sweet manufacturers, however, I am told that it is to the last degree improbable that these would contain phosphorus. I think it may fairly be said that the presence of phosphorus poisoning in this case is, at all events, "not proven." With regard to the question of alcoholic excitation, this in its turn may be also excluded. The patient was one of a teetotal family, belonged to a temperance society, and had never tasted alcohol in her life, according to her statement.

The case is obviously one to be recorded while waiting for further similar cases to throw a light on its exact nature. It is, of course, a matter for regret that the necropsy was so slightly and hurriedly performed; but I encountered the most strenuous opposition from the family.

Grays, Essex.

AN ExTRAORDINARY STORY OF WHOLESALE PoIsoning.-A story has reached us from Heath Town of wholesale poisoning by soup which in some of its particulars reminds us strongly of the Wylde Green cases which occurred during the Christmas week of last year. Nine persons, ranging in age from three to eighty-one, partook of some soup distributed in charity from the vicarage, and all afterwards showed symptoms of poisoning. In one sad case death ensued. 


\section{THE IMMUNISATION OF HORSES FOR THE PREPARATION OF DIPHTHERI ANTITOXIN. ${ }^{1}$}

BY WILLIAM ROBERTSON, M.R.C.V.S.,

ASSISTANT, VE'TERINARY DEPARTMENT, BRITIST INSIITUTE OF PREVLNTIVE MEDICINE.

IT is my intention to describe to the best of my ability the method employed in the immunisation of horses for the preparation of diphtheria antitoxin, and also to describe the process of bleeding these animals when immune. I find that suitable horses of the heavy draught class can be purchased for prices varying from $£ 310 \mathrm{~s}$. to $£ 7$. The animal is, in the first instance, placed in an isolation box, where it is tested with mallein (generally twice), then with tuberculin, and if the test is negative it is placed amongst the other horses. The seat of inoculation which I find most convenient is just in front of the shoulder; this part is easily accessible in the horse, and the operator is less liable to be kicked by the animal. Previously to inoculation the syringe \&c. are boiled for fifteen minutes and the part washer with ether and then with a solution of carbolic acid ( 1 in 20). The syringe, with needle affixed and charged with toxine, is then held in the right hand and a fold of sterilised skin pinched up with the left, the needle inserted, and the piston pushed gently home. As a rule the horse stands quietly enough and seldom exhibits any symptoms of pain or uneasiness during the inoculation. In commencing the immunisation of an animal very small quantities of the toxine are used at first, the initial dose being generally 1 c.c. In the case of the first horse experimented on I used toxine mixed with Gram's solution of iodine, but this method was soon discontinued, as it was found that the solution of iodine acted as an irritant and caused local swelling slow of absorption. The local lesion following the first inoculation is, as a rule, well marked and appears in the form of a diffuse soft swelling slightly painful on pressure. This swelling reaches its greatest height in from six to seven hours after inoculation, generally persists for two or three days, and then disappears. Coexistent with the local lesion we generally get a rise in temperature. This rise reaches its maximum in from six to seven hours after inoculation-that is to say, at the same time at which the local swelling is greatest-and generally falls to normal in thirty-six hours. When the local lesion has disappeared and the temperature fallen to normal the dose of toxine is repeated, and if this produces very little local or general effect it is raised by degrees, thus: primary dose, 1 c.c. ; then after a week has elapsed, 1 c.c. again; then 5 c.c.; then 10 c.c. ; then 10 c.c. again; then a rise to 25 c.c., and so on. When the dose has reached 50 c.c. we give the horse this quantity three times a week for a fortnight, and if the animal stands this amount without any reaction the dose is raised to 100 c.c. three times a week for the same length of time, and then 200 c.c. at the same intervals. The period of time allowed to elapse between each inoculation varies with different animals, and we take as a guirle the local swelling and the temperature, as the inoculations are discontinued until both have subsided.

The above is the outline of the method of immunising horses, but I have noticed a few facts in connexion with the above remarks which are of some interest, but which are of a somewhat disjointed nature. No two horses are alike in their power of resistance to the action of diphtheria toxine. I have had an animal under my charge which received 10 c.c. toxine, and the day after received 100 c.c., and this without producing any very marked effect; while in another case the initial dose of 1 c.c. produced a violent local and general reaction, and after an interval of a fortnight a second dose of 2 c.c. caused paralysis of the hind quarters and the animal had to be destroyed. The same toxine was used in both cases. Again, in some animals the local lesion in the neck disappears twice as fast as in others, using the same toxine in both cases. The rise in temperature following inoculation is also very variable. I have a record of a 1 A paper read before the Pathological Society of London on 1 A paper read
March 19th, 1895 . horse whose temperature during the whole process of immunisation never exceeded $103^{\circ} \%$, and I have a recors of another horse also immune who after every inosulation had a rise in temperature, in one case excecling $105^{\circ}$. Amongst all the horses I have inoculated, even when the temporature was very high, I have nevor known one to refuse its food; but I have noticed that following on a large desse of tozine some animals have a fit of shivering and exhibit distinct rigors. In a very few cases--four out of many hundreds of inoculations - I have seen necrosis of the skin to follow inoculation. The skin over the swelling becomes brown and harrt, and on falling off leaves a whazed red surface; this, however, soon heals uniler a boratic acid dressing. When the horse is considered to be snfliciently inmune it is allowed to rest for a clear week from the last inoculation before being bled. The vein from which wo bleed is the jugular vein found on the sirle of the inimal's neck lying in what is termed the jngular furrow and sepa rated from the skin by the panniculus carnosus, that wheet of muscular tissue stretching subcutaneously over the apper part of the horse's body. The part over the rein is first shaved and then washed thoroughly with ether and carbolic solution ( 1 in 20$)$. The vein is then raised by pressure with the fingers, and with a scalpel a small incision about half an inch in length is made through the skin and panniculus carnosus. ${ }^{2}$ On separating the edges of the wound with a pair of forceps the vein can be observed at the bottom of the incision. The cannula, after being connected to the Kistisato flask by a piece of quarter-inch rubber tubing, is tater in the right hand, the vessel is raised with the left and the tube inserted, care being taken that the stream of the vein is not interfered with. The blood then flows freely, and when the flask is sufficiently full it is replacer by an empty one as follows. The rubber tube is pinched by the fingers of the left hand and the full flask withdrawn and handed to an assistant to plug. 'The empty flask is now taken in the right hand, the long glass tube is placed in the end of the rubber piping, the constricting fingers are withdrawn, and the blood flows.

As regards the amount of blood which can be removen from an animal with impunity, that of course varies with size. The amount I generally take is ten litres, but I have removed as much as sixteen litres without any injurious effect to the horse; this is not to be wondered at, seeing that one-sixteenth part of the animal's bolly weight is blood The reason why I do not as a rule draw off more than ten. litres at a time is that if more is taken $I$ find the last four or five litres over the tenth do not clot properly, and the serum which separates is of a pink colour. As soon as the blood is firmly clotted it is advisable to twist the flask in the hand, so as to separate the clot from the walls. Wher. sufficient blood has been drawn off I press on the vein anterior to the camnula and withdraw the tube. I ther. sponge gently with carbolic solution (1 in 20) and fasten the edges of the wound with two catgut sutures. The wound is now dusted with iodoform and covered with a piece of sterilised wool dipped in collodion. I have never had any difficulty in stopping bleeding, and the wounds have always gone on satisfactorily. The veins are in no case obliterated. or obstructed, and in one horse which has been bled fourteer times the vessel is just as pervious as in any other animal.

It is hardly necessary for me to state that all instruments. \&c. are boiled for half an hour previously to an operation. As regards methods of restraint in this operation, these of course depend upon the animal, but I have usualky found horses stand quietly enough when being bled. I should like. to say a few words about the flasks in which the blood is collected and the method of decanting the serum. The flasks are ordinary Q-like filtering flasks fitted with a rnbber cork and two pieces of glass tubing, one of wide calibre to allow escape of air, the other of narrower dimensions, over the orifice of which is slipped the rubber tubing: connected to the canmula. These flasks are sterihised for three hours on three separate occasions. Wher the flasks are full and the blood has been twisted on: clotting they are placed in a cool cellar, and in twenty-four hours' time a large amount of serum will have separated. The branch tube of the flask is flamert in a spirit-lamp, the wool plug removed, and the scrum poured off into sterilised.

At first the cannula was inserted directly into the vein through the skin, but afterwards it was found to be more satisfactory to mats ins incision. 